\title{
EFECTO DE LA MATERIA ORGÁNICA Y EL MANEJO SOBRE LA HIDROFOBICIDAD DE SUELOS VOLCÁNICOS ${ }^{1}$
}

\author{
José Cuevas Becerra ${ }^{1,2}$ \\ ${ }^{1}$ Escuela de Graduados, Facultad de Ciencias Agrarias, Universidad Austral de Chile. \\ ${ }^{2}$ Instituto de Investigaciones Agropecuarias, INIA, Intihuasi. Covarrubias 185, Ovalle. \\ Correo electrónico: jcuevas@inia.cl
}

\section{Organic matter and management effect over hydrophobicity in volcanic ash soils}

Keywords: Soil porous system, hydrophobic soils, contact angle.

\begin{abstract}
Soil water repellence or hidrofobicity depends on a strong interaction among the mineral and organic soils fractions. The content of organic matter is an determinant factor of water repellence. A positive relationship between the content of organic matter and water repellence can be observed, however, not all organic matter may cause repellence in the soil matrix. More than the amount of organic compounds, the quality characteristics impact the hidrofobicity of soil. It is possible to find two types of repellencies:) natural and induced one. The repellence influence the magnitude of intermolecular electrical forces which act in the interface of solid-liquid-gas. This phenomenon can be evaluated by measuring the contact angle $(\theta)$ among these phases. Soil management influence water repellency, but this effect is reduced by intense mechanical labor. Hidrofobicity influence soil hydraulic characteristic, modifying preferential flows.

The aim of this study was: i) determine the soil water repellency in three volcanic soils from southern of Chile in combinations with two managements (forest and prairie) and ii) to estimate the buffer capacity by using a hydrophilic compound in the volcanic soils. It was observed different contact angle, attributed to soil characteristics, but with a direct effect of vegetation type which contributed to organic materials top cause repellence in these soils. Soils with similar pedogenetic characteristics show different hydrophobic values for the same vegetation (forest or pastures). Soils humectation capacity depends of the quality of soil organic matter more than SOM total content. Buffer capacity of soils depended of the amount of SOM, and its interaction between mineral soil particles.
\end{abstract}

Palabras clave: sistema poroso del suelo, suelos hidrófobos, ángulo de contacto.

${ }^{1}$ Parte de estos resultados fueron presentados en el X Congreso de la Ciencia del Suelo. Santiago, Noviembre de 2005. 


\section{RESUMEN}

La repelencia al agua o hidrofobicidad de los suelos depende de una fuerte interacción entre las fracciones minerales y orgánicas del suelo. El contenido de materia orgánica condicionara de manera importante el monto de la repelencia, pero no todo el Carbono orgánico es repelente, y mas que el monto total de compuestos orgánicos, son sus características traducidas como calidad de materia orgánica, la que incidirá sobre los montos de hidrofobicidad. Es posible encontrar dos tipos de repelencias, naturales e inducidas. La repelencia se origina por los cambios en las magnitudes de las fuerzas intermoleculares eléctricas que actúan en la interfase de sólido-liquido-gas pudiendo ser evaluada a través del ángulo de contacto $(\theta)$ entre estas fases. Existe una relación positiva entonces entre el contenido de materia orgánica y la repelencia. El manejo afecta la repelencia siendo esta menor cuando las solicitaciones mecánicas son mas intensas. La importancia de la hidrofobicidad es que cambia las características hidráulicas de los suelos, provocando la aparición de flujos preferenciales. Los objetivos de esta investigación, fueron determinar las variaciones en la repelencia al agua en tres suelos, originados a partir de depósitos de ceniza volcánica del sur de Chile bajo dos tipos de manejo (Bosque y Pradera) y el uso de un compuesto hidrofílico para estimar la capacidad tampón de los suelos a la hidrofobicidad. Se observó que los suelos analizados muestran valores del ángulo de contacto diferentes, que se atribuyen a las características propias del suelo, pero con un efecto directo del tipo de cubierta vegetal, que a través de aportes de materiales orgánicos y la interacción que se establezca con el suelo a nivel de agregados. Así suelos con igual material generador, muestran valores distintos de hidrofobicidad para condiciones de manejo y cubiertas vegetales similares. De manera que, la capacidad de humectación de los suelos depende en mayor medida de la calidad de materia orgánica que de su contenido total. Y la interacción entre las partículas de suelo y los componentes orgánicos.

\section{INTRODUCCIÓN}

La repelencia al agua o Hidrofobicidad de los suelos esta determinada por la interacción entre el contenido de materia orgánica y la textura del suelo. En general la repelencia incrementa con aumentos de la materia orgánica y disminuye con aumentos en los contenidos de arcilla y limo de los suelos (Harper et al., 2000). Así como existe una relación directa de la hidrofobicidad con la materia orgánica y la textura hay una relación aun más compleja entre la textura del suelo y los tipos de componentes orgánicos de este (Capriel et al., 1995).

La presencia de materia orgánica (MO) en el perfil de suelo es clave para la apari- ción de hidrofobicidad. Por lo que el contenido de carbono orgánico en el suelo permitiría predecir la repelencia, aunque en algunos casos solo una pequeña proporción de las variaciones en la hidrofobicidad puede ser explicada solo por las concentraciones totales de carbono orgánico (Harper et al., 2000). Relaciones multivariadas incluyendo carbono orgánico y las arcillas pueden explicar las variaciones en hidrofobicidad sin embargo, no todo el carbono es hidrofobico, de modo que una mejor relación entre la hidrofobicidad y el carbono orgánico puede obtenerse si se considera el tipo de carbono orgánico presente en el suelo que simplemente la cantidad total de este (Harper et al., 2000). Dentro de 
los compuestos que generan la hidrofobicidad tenemos compuestos alifáticos de cadena larga, alcoholes, esteres de ceras con cadenas extensas de polimetileno (Ma'shum et al., 1988). Además ácidos grasos, alcanos, fitoles, derivados de fitol, y esteroles han sido aislados desde suelos repelentes y asociados a la hidrofobicidad (Ma'shum et al., 1988).

Existen dos tipos de repelencia, natural e inducida. La repelencia natural depende de la textura, acidez y tipo de cobertura vegetal (Fabres, 2001). Además es posible observar repelencias bajo diferentes condiciones climáticas, describiéndose una estacionalidad natural que se hace evidente con la primera lluvia de verano, pero que desaparece con el aumento de las precipitaciones (Fabres, 2001).

La afinidad o repelencia de una superficie sólida con el agua se origina por las fuerzas en las interfases. Debido a las fuerzas intermoleculares de naturaleza eléctrica, éstas no sólo actúan entre moléculas de igual material (cohesión), sino que también entre moléculas de distintas características (adhesión). Si la atracción entre el agua y la superficie sólida es mayor que la cohesión interna de las moléculas de agua, el líquido se esparce sobre la superficie. Si esta atracción es menor, las partículas rechazan el agua y se forma un menisco cóncavo en el cual se observa un ángulo de contacto $(\theta)$ entre las tres fases (Tschapek, 1966).

Cuando el ángulo de contacto $(\theta)$ se encuentra entre $90^{\circ}$ y $0^{\circ}\left(90^{\circ} \geq \theta \geq 0^{\circ}\right)$ a la presión dentro del capilar, el material disperso absorbe agua; cuando, en el caso contrario, $\theta>90^{\circ}$, la presión anticapilar la hace impenetrable y se produce la repelencia. Ambas presiones están definidas por la ecuación de Laplace (1).

$$
\mathrm{P}=\gamma_{\mathrm{lg}}(1 / \mathrm{R} 1+1 / \mathrm{R} 2)=2 \gamma_{\mathrm{lg}} / \mathrm{R}=2 \gamma_{\mathrm{lg}} \operatorname{Cos} \theta / \mathrm{r}
$$

Donde $\mathbf{R}_{1}$ y $\mathbf{R}_{2}$ son el radio principal del menisco del líquido, $\mathrm{R}$ es el radio de la superficie de la esfera del líquido, $r$ es el radio del capilar y $\theta$.

Si la presencia de materia orgánica en el perfil de suelo es necesaria para la repelencia del agua, existiendo una relación positiva entre el carbono orgánico y la repelencia al agua. (McKissock et al., 2003). A medida que aumenta la intensidad en el laboreo del suelo, el ángulo de contacto disminuye y con ello aumenta la capacidad de humectación. Por otro lado la humectación decrece cuando existen aportes de materiales orgánicos en alta cantidad y frecuentes, como ocurre en suelos desarrollados bajo bosque. Un diferencial en la humectación de sitios con distinta cubierta vegetal y similar contenido de materia orgánica se puede explicar por diferencias cualitativas en la materia orgánica. (Ellies et al., 1996).

Existe una estrecha relación entre la estabilidad de los agregados y el potencial de humectación. Los suelos cuyo manejo mantiene la hidrofobicidad, presentan agregados estables a la dispersión y por ende, muestran pequeñas variaciones del diámetro medio ponderado de los agregados. Así los agregados en sitios más hidrófobos son más finos (Ellies et al., 1996).

Es posible afirmar que la disminución de materia orgánica tiene efectos detrimentales sobre las propiedades físico mecánicas del suelo, especialmente por una disminución en las capacidades de retención de humedad, que estarían explicadas por este recubrimiento de componentes orgánicos de los agregados del suelo. Si se rompe este continuo de materiales orgánicos sobre la superficie, también se observa una disminución de la resistencia a la humectación de los suelos, y las resistencias mecánicas de los agregados se regirán por fuerzas mecánicas como el ángulo de roce entre las partículas.

La importancia de la hidrofobicidad, es 
que cambia las propiedades hídricas de los suelos. La capacidad de retención de humedad es afectada significativamente por cambios en la energía entre las fases sólida, líquida y gaseosa (Robichaud y Hungerford, 2000). Una repelencia extrema puede llevar a una perdida completa en la capacidad de infiltración de un suelo, favoreciendo la erosión y la ocurrencia de deslizamientos de terrenos (Robichaud y Hungerford, 2000). La hidrofobicidad además favorece la aparición de flujos preferenciales, con los consiguientes patrones de humectación desuniformes (Dekker et al., 2001). Brown (1987) sugirió que los escurrimientos superficiales en respuesta a lluvias cerca del lago Tahoe (USA) estaban asociados a esta relación de flujos preferenciales e hidrofobicidad, encontrándose evidencia para afirmar que los efectos de la repelencia sobre los flujos preferenciales podrían ser temporales y aun que los flujos preferenciales podrían disiparse después de exposiciones prolongadas a humedad (ejemplo durante la fusión en primavera de la capa de nieve) (Burcar et al., 1994). Esto por la existencia de dos escalas de flujo preferencial (Luxmoore 1991, citado por Burcar et al., 1994), la primera a través de los macroporos del suelo, con una baja superficie específica por unidad de volumen lo que permite una rápida infiltración y tasa de transferencia de agua, esto genera que la matriz de suelo tenga poca influencia en la calidad del agua que infiltra. Y una segunda escala a nivel de los poros medios y finos, con una mayor superficie por unidad de volumen y un lento flujo de agua, permitiendo una gran interacción entre sólido y líquido.

En el caso de los suelos de la zona sur de Chile, esto podría explicar las altas tasas de infiltración aun en suelos con altos contenidos de materia orgánica y por lo tanto de materiales hidrofóbicos, ya que la repelencia tendría su efecto en los primeros instantes de la lluvia, en la que se produce el mayor escurrimiento superficial y los mayores montos de arrastre de materiales, aunque una vez que el suelo se ha saturado comienza a funcionar el sistema poroso del suelo, y el agua comienza a infiltrar.

Se plantea que en suelos de ceniza volcánica bajo sistemas de manejo diferentes existirán niveles de repelencia dependientes de aspectos cualitativos de la materia orgánica, y que estos aspectos pueden ser visualizados a través de la resistencia al cambio en la hidrofobicidad en diferentes suelos, medida a través de la variación en el ángulo de contacto.

Los objetivos de este trabajo fueron medir el valor del ángulo de contacto en suelos de ceniza volcánica con diferentes grados de evolución, bajo diferentes intensidades de manejo. También se evaluó la medición del ángulo de contacto y el uso de materiales hidrofílicos (en este caso cuarzo) para estimar el grado de resistencia al cambio en la capacidad de humectación de los suelos.

\section{MATERIALES Y MÉTODOS}

Los análisis se realizaron en el laboratorio de Física de Suelos del Instituto de Ingeniería Agraria y Suelos de la UACH. Los suelos utilizados corresponden a serie Pemehue (Hapludands, USDA, 1998) ubicada en la Provincia de Cautín; perteneciente a la Novena Región. De la Décima Región se seleccionaron las Series Fresia (Palehumult, USDA, 1998), ubicado en la Provincia de Llanquihue y la Serie Osorno (Hapludands, USDA, 1998) que se ubica en la provincia de Osorno.

Para cada suelo se seleccionaron dos sectores, uno con manejo de bosque y otro de pradera. De cada sitio se extrajeron muestras de los primeros $10 \mathrm{~cm}$. de suelo.

Los suelos fueron tamizados 38 y $63 \mu \mathrm{m}$. Una submuestra monogranular de 
microagregados fue depositada uniformemente sobre un portaobjetos de vidrio. Los gránulos fueron adheridos al portaobjeto mediante una cinta adhesiva de doble contacto. Sobre los microagregados se colocaron 10-12 gotas de agua de $0,02 \mathrm{~cm} 3$ saturada con $\mathrm{KCl}$, volumen que fue adicionado mediante una jeringa con un micrómetro. Cuando la gota es colocada sobre una superficie hidrofóbica o parcialmente humectable esta no se esparce completamente sobre esta superficie. La gota asume una forma que dependerá de la relación entre la energía libre de las tres fases envueltas en el proceso; líquido-vapor de agua $\left(\boldsymbol{\sigma}_{\mathrm{lv}}\right)$, sólido-vapor de agua $\left(\boldsymbol{\sigma}_{\mathrm{sv}}\right)$ y sólido-líquido $(\boldsymbol{\sigma}$ sl). Y la relación entre ellas fue definida por Young como (2):

$$
\sigma_{\mathrm{lv}} \cos \theta=\sigma_{\mathrm{sv}}-\sigma \mathrm{sl}
$$

Paralelamente se procedió a moler cuarzo y posteriormente tamizado dentro del mismo rango, 38 y $63 \mu \mathrm{m}$. Este fue mezclado con el suelo tamizado en proporciones de $3 \%$ de arena de cuarzo, $5 \%$ de cuarzo y $10 \%$ de cuarzo base volumen. Para evaluar el efecto de la adición del cuarzo, se midió el ángulo de humectación según la metodología propuesta por Contreras (2000).

Las observaciones de la forma de la gota se realizaron con una lupa provista de un campo de visión horizontal. El ocular de esta lupa presenta un goniómetro que permite medir el ángulo que se forma entre la gota de agua depositada sobre una superficie y esta superficie (Burckhardt, 1985 citado por Ellies, 2003). Bachmann (1998), describe esta metodología donde propone adherir el material a un portaobjetos mediante una cinta adhesiva de doble contacto, de esta manera se disminuye el efecto de la rugosidad del suelo.

El contenido de materia orgánica de los suelos se determinó con una combustión húmeda (Sadzawka et al., 2004).

Se realizó análisis estadístico multivariado, con prueba de Tuckey al 5\%, a través del Software statgraphic plus v 2.0.

\section{RESULTADOS Y DISCUSIÓN}

\section{Contenido de materia orgánica del Suelo}

El Cuadro 1 presenta los contenidos de materia orgánica de la primera estrata de los suelos analizados, los valores obtenidos son concordantes con datos obtenidos en otras investigaciones en estos suelos. Aunque no presentan diferencias estadísticamente significativas, existe una tendencia a valores mayores de los contenidos de materia orgánica para el bosque con respecto a la pradera, lo que es lógico si se considera el grado de intervención que tiene cada uno de los sitios analizados, en el bosque las adiciones de materiales orgánicos son mas constantes que bajo pradera. Se deben considerar otros factores del sitio que difieren para el bosque y para la pradera como las tasas de mineralización, el pH, las constantes hídricas bajo bosque que difieren de las del suelo bajo pradera. Además el material orgánico es de naturaleza diferente, según Ellies et al. (1996), bajo bosque es posible encontrar componentes orgánicos de cadenas mas largas que muestran propiedades hidrófobas mayores que las encontradas bajo la pradera, lo que permite hablar de "calidad" de materia orgánica desde el punto de vista de la hidrofobicidad. 
Cuadro 1: Contenido de materia orgánica en los suelos estudiados.

Table 1: Soil Organic matter content in the studied soils.

\begin{tabular}{cccc}
\hline Suelo & Manejo & Profundidad & \% M.O.S. \\
\hline \multirow{2}{*}{ Osorno } & Pradera & $0-10 \mathrm{~cm}$. & 15.38 \\
\cline { 2 - 4 } & Bosque & $0-10 \mathrm{~cm}$. & 26.33 \\
\hline \multirow{2}{*}{ Fresia } & Pradera & $0-10 \mathrm{~cm}$. & 12.59 \\
\cline { 2 - 4 } & Bosque & $0-10 \mathrm{~cm}$. & 14.5 \\
\hline \multirow{2}{*}{ Pemehue } & Pradera & $0-10 \mathrm{~cm}$. & 16.03 \\
\cline { 2 - 4 } & Bosque & $0-10 \mathrm{~cm}$. & 17.76 \\
\hline
\end{tabular}

\section{Variación de la repelencia}

En la Figura 1 están graficados los valores de $\theta$ para los suelos bajo investigación en función del manejo. Se observan diferencias entre los valores encontrados en el bosque y la pradera, lo que concuerda con lo establecido por Ellies et al. (1996). Los valores encontrados son menores a los expresados en la literatura acerca de la clasificación de suelos hidrofóbicos $\left(\theta>90^{\circ}\right)$. Debe considerarse que esta metodología es la que provoca una mayor disturbación del suelo, sin embargo los valores obtenidos reflejan las diferencias en el grado de repelencia de estos. Los suelos estudiados presentan para la condición de bosque valores de $\theta$ sobre $70^{\circ}$ y en la pradera bajo $60^{\circ}$ en promedio. Todos los valores representan el promedio de 10 repeticiones obtenidas para todas las mediciones de ángulos de contacto. Las diferencias obtenidas coinciden con estudios realizados por Ellies et al. (1995), Ellies et al. (1996) y Ellies et al. (2003) para suelos volcánicos. En el caso del bosque se puede inferir que las sustancias que repelen al agua provienen de adiciones recientes de $\mathrm{MO}$ o que corresponden a estados primarios de descomposición. Sitios con una baja capacidad de humectación presentan un alto tenor de materia orgánica y en Chile se encuentran bajo bosque nativo o en suelos poco intervenidos, debido a que el uso del suelo influye en las propiedades de la materia orgánica en dos formas: alterando el aporte anual que procede después de la muerte de plantas y animales y variando el ritmo con que ésta se mineraliza (Russel, 1992). Esto resulta en una menor perdida de $\mathrm{MO}$ en el bosque que la pradera. 


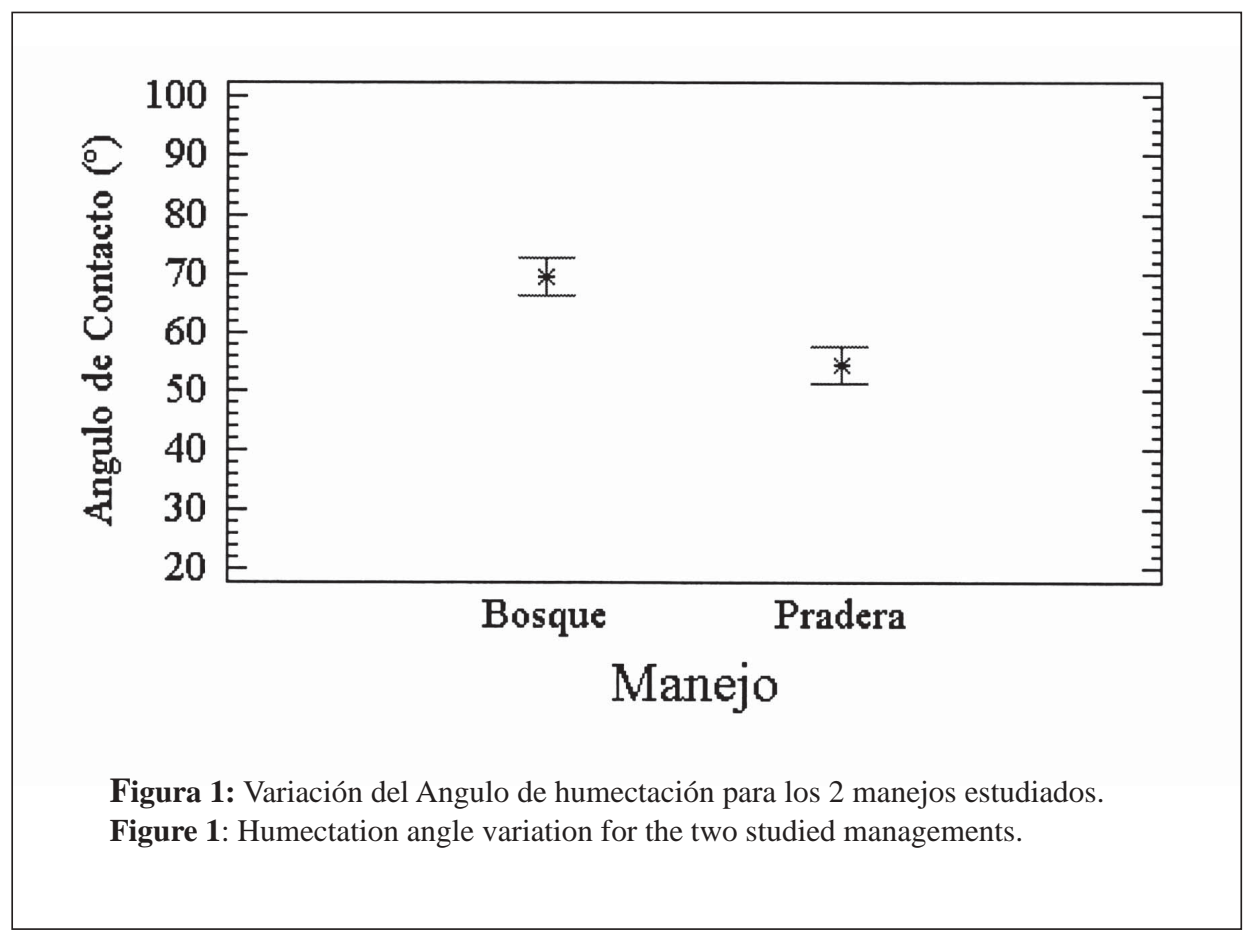

La Figura 2 muestra la variación del grado de repelencia a través de los valores medidos del ángulo de contacto en función del suelo y su contenido de materia orgánica. El contenido de MO no muestra diferencias para los tres suelos analizados, aunque existe una tendencia a mayores valores para el caso de Osorno dado por el alto contenido de $\mathrm{MO}$ en el bosque. Los valores de $\theta$ muestran una variación en función del desarrollo de los suelos, siendo menor para el Palehumult y mayores en los Hapludans analizados. La Serie Fresia presenta una humectación muy similar a Osorno. En los Hapludands, la Serie Pemehue presenta la más baja humectación con los valores de ángulo de contacto más altos, seguido por la Serie Osorno. Ellies (1975), señala que en Hapludands de textura más gruesa, la hidrofobicidad es más acentuada que en suelos de texturas más finas, esto explicaría los mayores ángulos de contacto en la serie Pemehue. Además este comportamiento está relacionado con el desarrollo pedogenético, donde un suelo menos evolucionado presenta una mayor repelencia al agua, debido a un aporte de cenizas más recientes o frecuentes que un suelo que se encuentra más alejado del cordón cordillerano con una mayor capacidad de humectación. Asociado a una mayor presencia de propiedades ándicas en los suelos, en los cuales el predominio de arcillas no cristalinas, como el alofán, favorece una mayor acumulación de materia orgánica, debido a la formación de complejos órgano-minerales que la estabilizan (Zunino y Borie, 1985; Tan, 1993). 


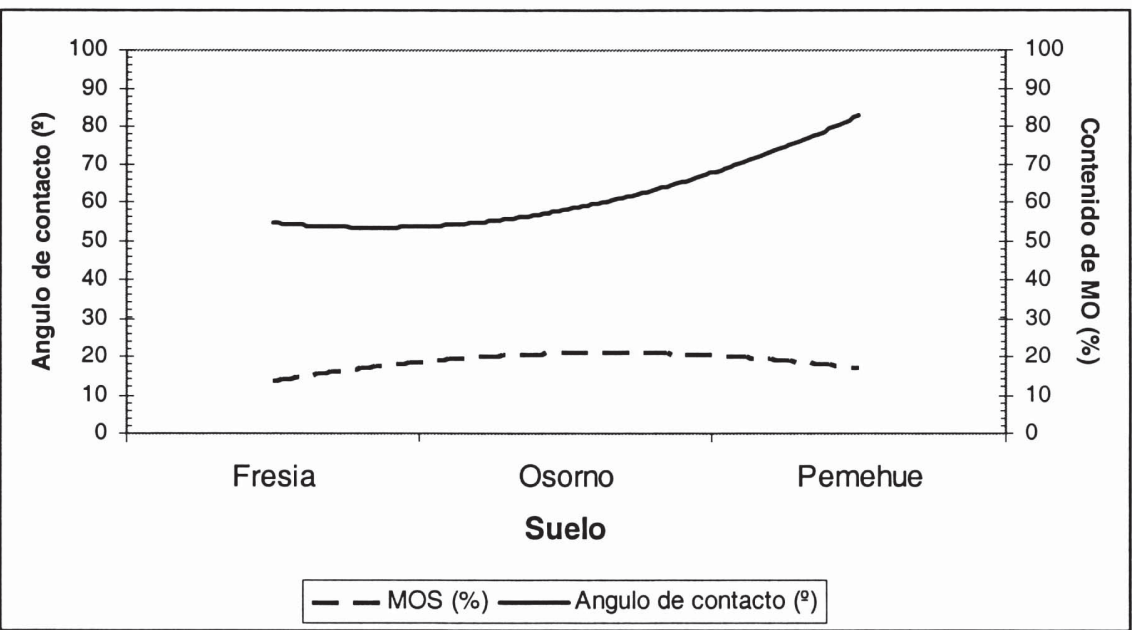

Figura 2: Variación del Angulo de contacto en función del tipo de suelo y su contenido de materia orgánica.

Figure 2: Contact Angle variation related to the soil type and organic matter content.

\section{Suelo Fresia}

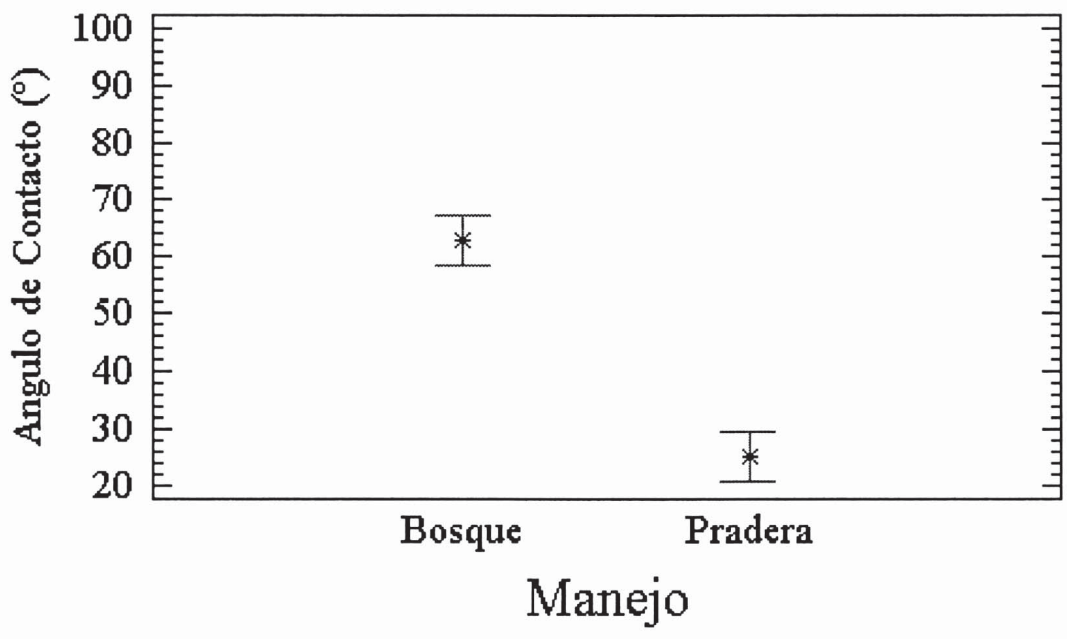

Figura 3: Ángulos de contacto medidos en el suelo Fresia, para 2 condiciones de manejo.

Figure 3: Contact angles assessed for Fresia soil, for two managements conditions. 
La Figura 3 muestra los valores obtenidos en el Palehumult. Acá los valores medidos son mayores en el bosque que en la pradera. Las diferencias son significativas, sobre $60^{\circ}$ en el bosque y menos de $30^{\circ}$ en la pradera. Al comparar estos valores con los contenidos de MO para ambos sitios (Cuadro 1) se observa que no hay diferencias entre ellos. Por lo que la tendencia a una mayor humectabilidad del Palehumult bajo pradera estaría dada tanto por la calidad de presentes a nivel de la matriz y de los agregados del suelo (Ellies et al., 2003).

En la figura 4 se observan los valores obtenidos para el Osorno. La pradera muestra valores de $\theta$ superiores al bosque. En este último los valores son cercanos a $60^{\circ} \mathrm{y}$ en la pradera sobre $70^{\circ}$. Si se comparan con los contenidos de $\mathrm{MO}$ (Cuadro 1) no existiría relación entre estos valores. Una posible explicación tendría que ver primero con la calidad del material orgánico encontrado

\section{Suelo Osorno}

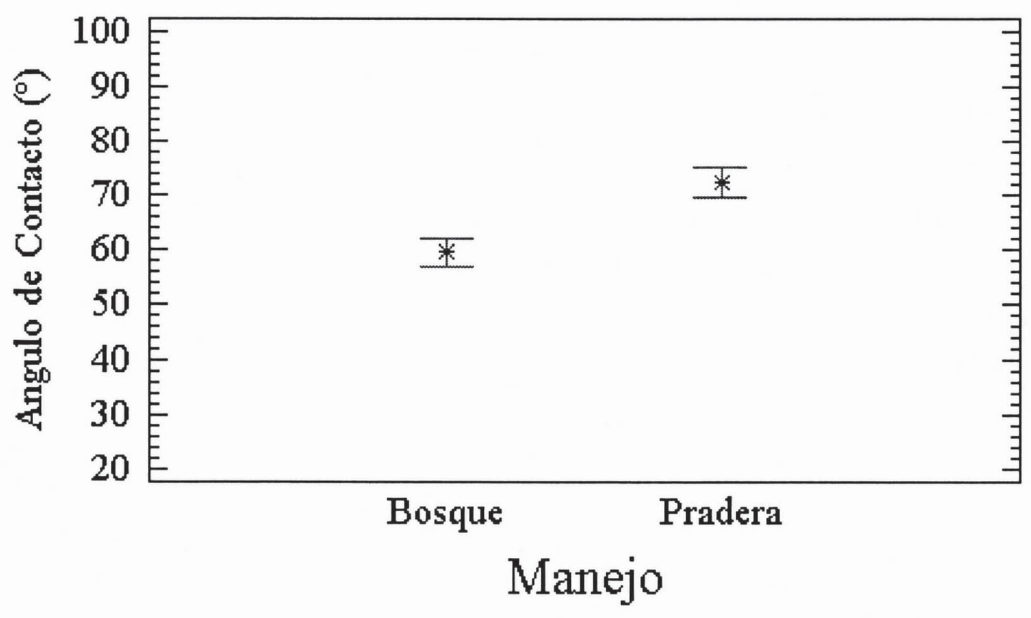

Figura 4: Ángulos de contacto medidos en el suelo Osorno, para 2 condiciones de manejo.

Figure 4: Contact angle measured to Osorno soil, for two management's conditions.

la materia orgánica, y del grado de intervención (solicitación mecánica) mayor en la pradera. Esta solicitación mecánica provoca una pulverización de los agregados, lo que se suma al hecho de presentar una granulometría fina y altos contenidos de arcillas, por lo tanto con una mayor superficie específica. Todas las variaciones en $\theta$ para este suelo estarán relacionadas con las características de los materiales orgánicos en ambos sectores. Con el aporte constante de materiales orgánicos provenientes del pastoreo directo en la pradera a través de las fecas animales, y finalmente con que el bosque ha sido intervenido en ocasiones para realizar raleos forestales, lo que ha modificado las condiciones de la MO del suelo en este sitio, esto es importante ya que este suelo muestra valores bajos para los encontrados por Ellies et al. (1996). 


\section{Suelo Pemehue}

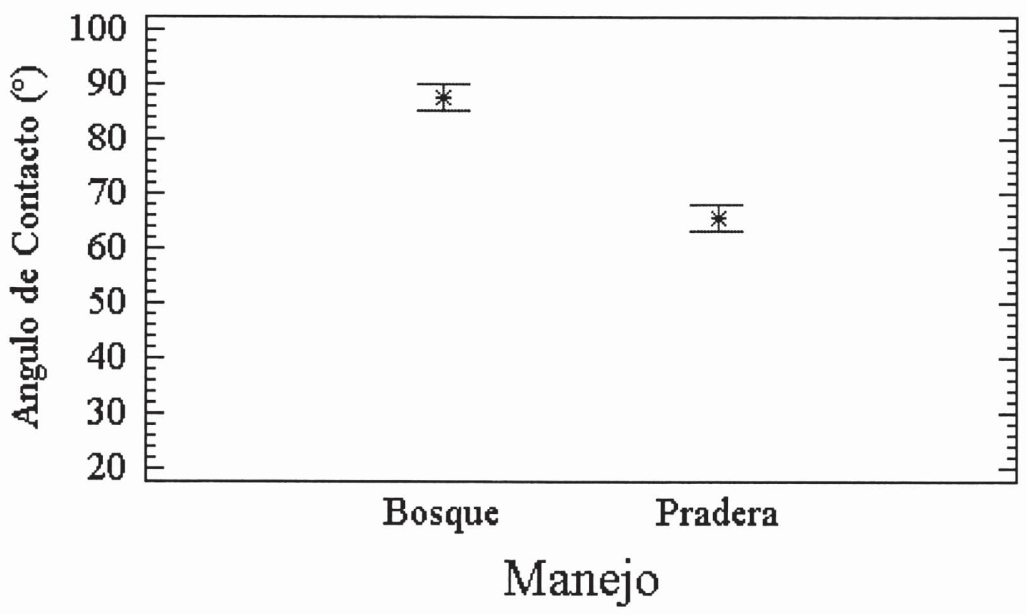

Figura 5: Ángulos de contacto medidos en el suelo Pemehue, para 2 condiciones de manejo.

Figure 5: Contact angle measured in Pemehue soil, under two management conditions.

En el caso del Pemehue (Figura 5), este no se comporta de igual manera que el antes analizado. Los valores para el bosque de $\theta$ son cercanos a $90^{\circ}$ lo que indica hidrofobicidad. La pradera muestra valores cercanos a $60^{\circ}$. Los valores de MO prácticamente no difieren entre ambos sitios. Según Nissen et al. (2006) este es un suelo menos evolucionado que los anteriores, mostrando un menor numero de poros, por lo que la superficie en la cual actuará la materia orgánica es menor que para Andisoles mas desarrollados como es el caso del Osorno. En este caso las diferencias en la repelencia estarán también dados por el tipo de MO presente en el suelo, lo que concuerda con lo expuesto por Ellies et al.(1995), quienes señalan que la humectación de los suelos tiende a disminuir cuando los aportes orgánicos son altos y frecuentes, lo que es mas factible de ocurrir bajo una vegetación boscosa.

\section{Resistencia al cambio en la repelencia}

La Figura 6 muestra el cambio en los valores de $\theta$ al adicionar algunas cantidades de gránulos de cuarzo de entre 38 y $63 \mu \mathrm{m}$ para el suelo Fresia. En el caso del bosque el valor del ángulo de contacto disminuye desde cercano a $60^{\circ}$ hasta casi $10^{\circ}$ con aplicaciones de $10 \%$ de un material hidrofilico como el cuarzo. En el bosque la curva muestra una conducta distinta, con $3 \%$ de cuarzo aumenta el valor del ángulo hasta esta disminución es desde cerca de $70^{\circ}$ hasta $60^{\circ}$. La tendencia que se observa en la curva en el caso del bosque es a mantener los valores de $\theta$ y en la pradera a disminuir los valores de $\theta$ con aplicaciones de cuarzo. En este suelo en particular, la fuerza que opone al cambio en los valores del ángulo de contacto es mayor para el caso del bosque 
con respecto a la pradera, esto indica que si bien, las cantidades de materia orgánica no son estadísticamente diferentes, el comportamiento del suelo con respecto a calidades diferentes de MO si lo es. El suelo bajo bosque necesita una mayor cantidad de arena $\theta$ lo que esta de acuerdo con lo expresado por Ellies et al. (1996) y Ellies et al. (1995). Existe además un alto grado de interacción entre la MO y la superficie específica sobre la que esta actuando, ya que el suelo muestra una elevada capacidad tampón en el cam-

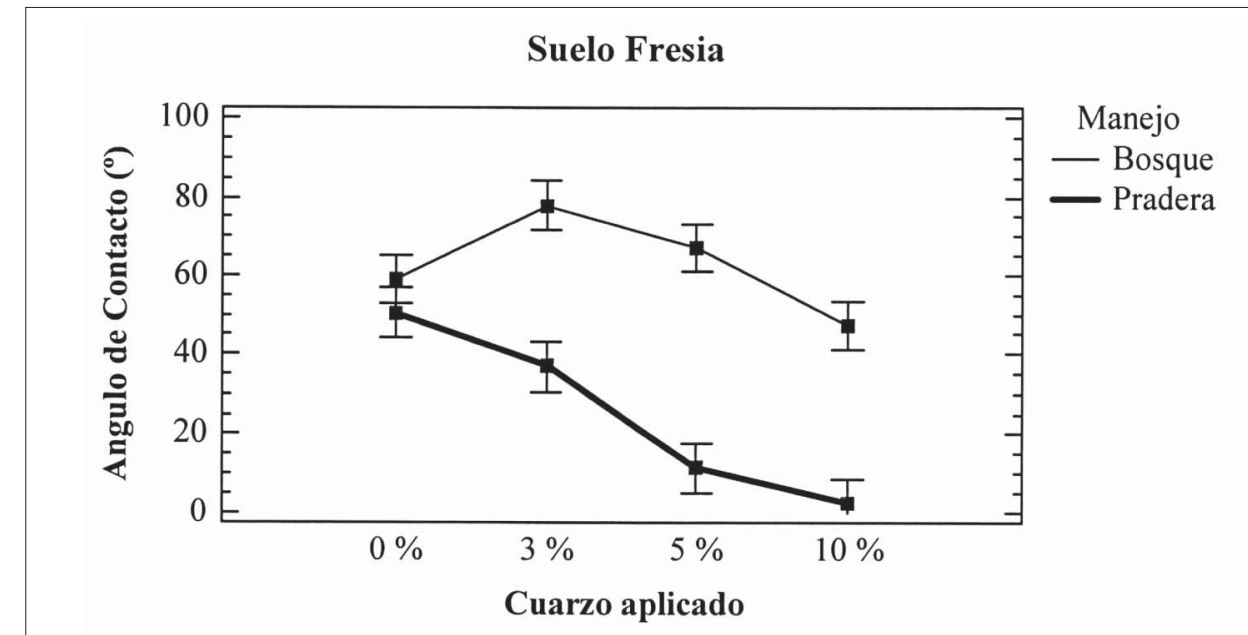

Figura 6: Variación del ángulo de contacto con aplicaciones de arena de cuarzo, para el suelo Fresia.

Figure 6: Contact angle variation with quartz sand applications, for Fresia Soil.

de cuarzo para provocar un cambio significativo en la capacidad de humectación, al revés de lo que pasa en la pradera, en que a iguales cantidades de materiales hidrofilicos aplicados, el cambio se produce en mayor magnitud y en menor tiempo.

Para la serie Osorno (Figura 7), al aplicar cantidades crecientes de cuarzo hay un aumento de los valores de $\theta$, llegando en la pradera hasta $75^{\circ}$ y en el bosque hasta $65^{\circ}$. El material hidrofilico agregado no fue capaz de interactuar a nivel de los gránulos de suelo. Esto se asocia a un manejo degradante, en que el pisoteo a que esta siendo sometido este suelo cambia las propiedades físicas del mismo modificando la microagregación. Los aspectos cualitativos de la MO tienen un efecto importante sobre bio en los valores de capacidad de humectación al aplicar un elemento hidrofílico como el cuarzo.

El Pemehue muestra una alta capacidad tampón para los efectos de arena de cuarzo (Figura 8), ya que se mantienen altos valores de $\theta$ con aplicaciones de cuarzo crecientes. En el bosque disminuye desde $90^{\circ}$ hasta $80^{\circ}$ y en el caso de pradera baja desde $70^{\circ}$ hasta $60^{\circ}$. Aunque la tendencia es a disminuir la repelencia, la pendiente de la curva muestra un comportamiento similar en ambos manejos. Las características de los materiales orgánicos presentes en ambos sitios tienen una alta estabilidad y una alta repelencia al agua, esto es interesante ya que podría indicar que las dinámicas de $\mathrm{MO}$, dependerían además del grado de evolución 

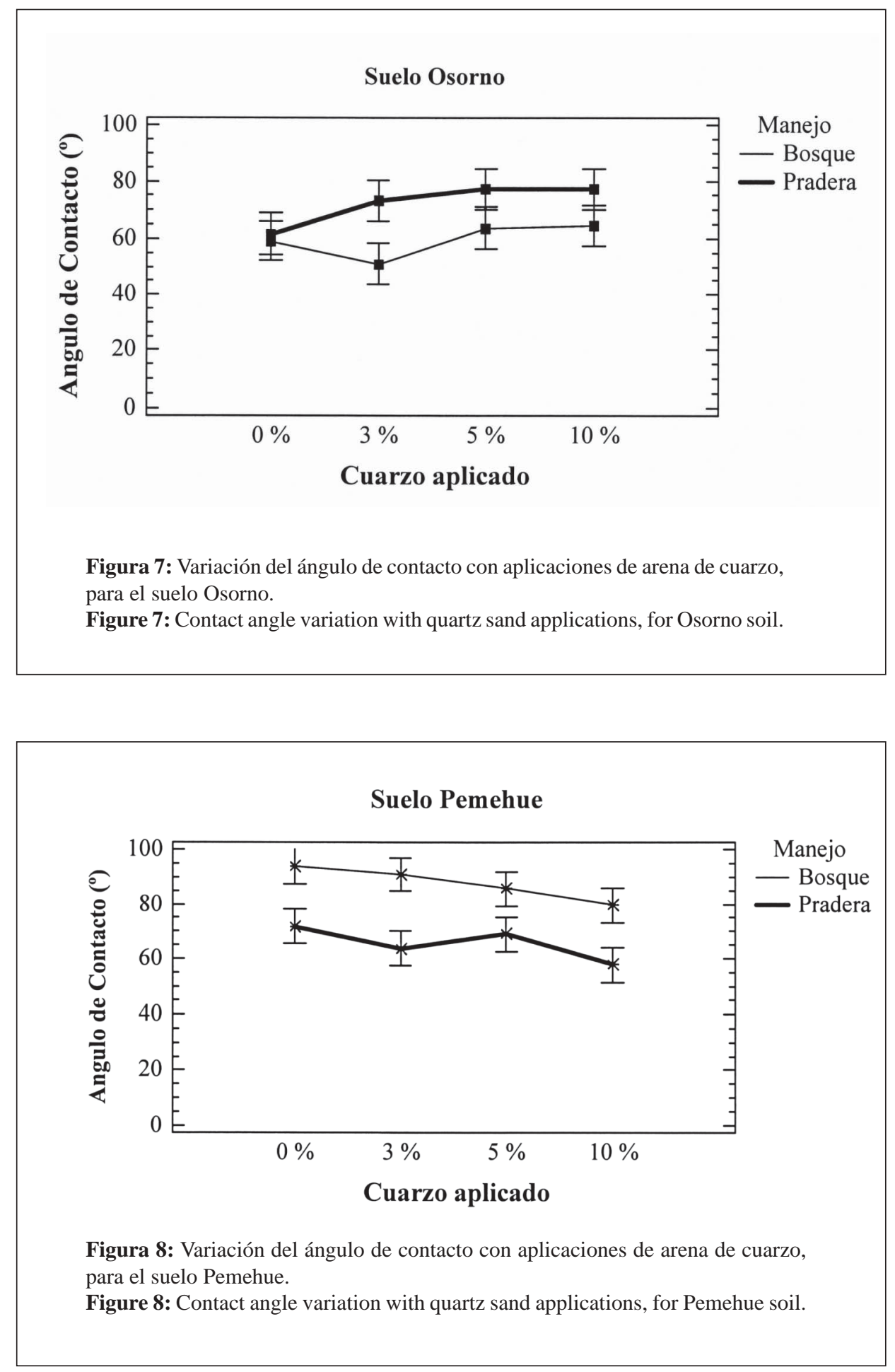
del suelo, del tipo de material orgánico y sus características hidrofóbicas de tipo cualitativo y en algunos casos cuantitativo junto con la superficie específica juegan un rol fundamental en la capacidad de humectación de estos suelos. Esta relación puede tener un efecto sinérgico generando sectores con hidrofobicidad. Se demuestra que la repelencia esta directamente asociada con el grado de descomposición de los materiales orgánicos del suelo, lo que permite inferir que los patrones de repelencia en la superficie del suelo y los primeros cm. mostrarán variaciones estacionales. Estas variaciones serán dependientes de los montos de materiales orgánicos aportados y el grado de descomposición de estos en función de las características climáticas, de la intensidad de manejo, y el suelo del sector analizado. Esto podría estar directamente relacionado con algunas fracciones de $\mathrm{MO}$, por ejemplo Fracción ligera. Este método pudiera servir como un índice para evaluar ésta en el tiempo a través del cambio en la capacidad de humectación en el tiempo.

\section{CONCLUSIONES}

Todos los suelos exhiben un cierto nivel de repelencia al agua o baja capacidad de humectación, lo que tiene efectos positivos o negativos. La repelencia puede ser medida a partir del valor del ángulo de contacto en la interfase entre el suelo y el agua. Este índice se denomina hidrofobicidad de los suelos que tiene su origen en características pro- pias del material, así como por efectos del manejo del suelo, o por especies vegetales presentes en una determinada zona. La hidrofobicidad esta en directa relación con los contenidos de materia orgánica del suelo. Es posible precisar que el valor del ángulo de humectación está dado por las características de la materia orgánica del suelo como cantidad, posición y especialmente la 'calidad' de la materia orgánica, entendida como el tipo de material del cual se origina, condicionado por el tipo de cubierta vegetal y el manejo que vía mayor o menor tasa de mineralización reflejará un mayor o menor grado de descomposición. La modificación de la repelencia muestra una capacidad tampón que es dependiente de las características antes mencionadas. En este estudio queda de manifiesto que para suelos de ceniza volcánica más que la cantidad total de materia orgánica del suelo, es el tipo o calidad de material orgánico y la posición estratégica en que se encuentre a nivel del sistema poroso en el suelo lo que incidirá sobre el grado de hidrofobicidad de los suelos. Y que el uso de gránulos de cuarzo puede ser un buen estimador de la resistencia al cambio en el valor de hidrofobicidad.

\section{AGRADECIMIENTOS}

En memoria al Dr. Achim Ellies S., fallecido en Septiembre 2004. Investigación financiada por Proyecto. FONDECYT 1040101. 


\section{BIBLIOGRAFÍA}

BACHMANN, J. 1998. A rapid method to measure water repellency of soils. $16^{\circ}$ Congreso Mundial de la Ciencia del Suelo, Montepellier, Francia. Summaries, Volumen I: 20 p.

BROWN, D. 1987 Nitrate cycling and hydrologic transport mechanism in a Sierra Nevada headwaters watershed. M. S. Thesis. Nevada University, Reno.

BURCAR, S.; MILLER, W.; TYLER, W., JONSON, D. 1994 Seasonal preferential flow in two Sierra Nevada soils under forested and meadow cover. Soil Sci. Soc. Am. J. 58:1555 - 1561.

CAPRIEL, P., BECK, T., BORCHERT, H., GRONHOLZ, J., ZACHMANN, G., 1995. Hydrophobicity of the organic matter in arable soils. Soil Biol. Biochem. 27, 1453e1458.

CONTRERAS, C. 1998. Metodología para evaluar la capacidad de humectación en suelos. Tesis Lic. Agr. Valdivia. Universidad Austral de Chile. Facultad de Ciencias Agrarias. 48 p.

DEKKER. L.; DOERR, S.; OOSTINDIE, K.; ZIOGAS, A. y RITSEMA, C. 2001. Water repellency and critical soil water content in a dune sand. Soil Science Society of America Journal.65: 1667-1674

ELLIES, A. 1975. Untersuchungen über einige Aspekte des Wasserhaushaltes vulkanischer Aschenböden aus der gemässigten Zone Südchiles. Disertation. Hannover, Technische Universität Hanover. Alemania. 107 p.

ELLIES, A., GREZ, R., RAMÍREZ, C. 1996. Efecto de la materia orgánica sobre la capacidad de humectación y as propiedades estructurales de alsunos suelos de la zona centro sur de Chile. Agro Sur 24(1):48-58.
ELLIES, A., GREZ, R., RAMIREZ, C. 1995 Potencial de humectación y estabilidad estructural de suelos sometidos a diferentes manejos. Agricultura técnica 55 (3-4): 220-225.

ELLIES, A., RAMIREZ, C., Mac DONALD, R. 2003 Wetting capacity distribution in aggregates from soils with a different management. Food, Agriculture \& Environment 1(2): 229-233.

FABRES, T. 2001 Variación de la humectación de los suelos según intensidad de uso y propiedades de la materia orgánica. Tesis Lic. Agr. Valdivia. Universidad Austral de Chile. Facultad de Ciencias Agrarias. 66 p.

HARPER, R., MCKISSOCK, I., GILKES, R., CARTER, D., BLACKWELL, P. 2000 A multivariate framework for interpreting the effects of soil properties, soil mangement and landuse on water repellency, Journal of Hydrology, 231-232: pp 371-383.

MA'SHUM, M., TATE, M., JONES, G., OADES, J. 1988 Extraction and characterization of water-repellent material from Australian soils. Journal of Soil Science 39, 99-110.

McKISSOCK, I., GILKES, R., Van BRONSWIJK, W. 2003. The relationship of soil water repellency to aliphatic $\mathrm{C}$ and Kaolin measured using DRIFT. Aust. J. Soil Res. 41: 251-265.

NISSEN, J., QUIROZ, C., SEGUEL, O., MAC DONALD, R., ELLIES, A. 2006 Flujo hídrico no saturado en Andisoles. R.C. Suelo Nutr. Veg., abr. 2006, Vol. 6, No. 1, pp. 9-19.

ROBICHAUD, P., HUNGERFORD, R. 2000. Water repellency by laboratory burning of four northern Rocky Mountain forest soils. Journal of Hydrology 231-232: 207-219. 
RUSSEL, A. 1992. Condiciones del suelo y desarrollo de las plantas según Russel. Madrid, Mundi-Prensa. España. $632 \mathrm{p}$.

TAN, K. 1993. Principles of soil chemistry. 2th. New York, Marcel Dekker, Inc. United States of America. 362p.

TSCHAPEK, M. 1966. El agua del suelo. Nuevas gráficas. Madrid, España. $480 \mathrm{p}$.

TSCHAPEK, M. 1984 Criteria for determining the hydrophilicity hydrophobicity of soils. Zeitschrift für pflanzenernährung und bodenkunde. 147: 137 - 149.
SADZAWKA, A., CARRASCO, M.A., GREZ, R., MORA, M.L. 2004a. Métodos de análisis recomendados para los suelos chilenos. Comisión de Normalización y Acreditación, Sociedad Chilena de la Ciencia del Suelo. 113 p.

SOIL SURVEY STAFF (SSS). 1998. Keys to soil taxonomy. 8th Ed. $326 \mathrm{p}$.

ZUNINO, H. Y BORIE, F. 1985. Materia orgánica y procesos biológicos en suelos alofánicos. In: Tosso, J.(ed). Suelos volcánicos de Chile. Instituto de Investigaciones Agropecuarias (INIA), Santiago. 5: 341-425. 